\title{
Stress Decreases Host Viral Resistance and Increases Covid Susceptibility in Embryonic Stem Cells
}

\author{
Mohammed Abdulhasan $^{1,2} \cdot$ Ximena Ruden $^{1} \cdot$ Benjamin Rappolee $^{3} \cdot$ Sudipta Dutta $^{1,4} \cdot$ Katherine Gurdziel $^{5}$. \\ Douglas M. Ruden ${ }^{1,6}$. Awoniyi O Awonuga ${ }^{1}$ - Steve J. Korzeniewski ${ }^{6}$. Elizabeth E. Puscheck ${ }^{1,2,8}$. \\ Daniel A. Rappolee ${ }^{1,2,6,7,9,10}$
}

Accepted: 13 May 2021 / Published online: 21 June 2021

(c) The Author(s), under exclusive licence to Springer Science+Business Media, LLC, part of Springer Nature 2021

\begin{abstract}
Stress-induced changes in viral receptor and susceptibility gene expression were measured in embryonic stem cells (ESC) and differentiated progeny. Rex1 promoter-Red Fluorescence Protein reporter ESC were tested by RNAseq after 72hr exposures to control stress hyperosmotic sorbitol under stemness culture (NS) to quantify stress-forced differentiation (SFD) transcriptomic programs. Control ESC cultured with stemness factor removal produced normal differentiation (ND). Bulk RNAseq transcriptomic analysis showed significant upregulation of two genes involved in Covid-19 cell uptake, Vimentin (VIM) and Transmembrane Serine Protease 2 (TMPRSS2). SFD increased the hepatitis A virus receptor (Havcr1) and the transplacental Herpes simplex 1 (HSV1) virus receptor (Pvrl1) compared with ESC undergoing ND. Several other coronavirus receptors, Glutamyl Aminopeptidase (ENPEP) and Dipeptidyl Peptidase 4 (DPP4) were upregulated significantly in SFD $>$ ND. Although stressed ESC are more susceptible to infection due to increased expression of viral receptors and decreased resistance, the necessary Covid-19 receptor, angiotensin converting enzyme (ACE)2, was not expressed in our experiments. TMPRSS2, ENPEP, and DPP4 mediate Coronavirus uptake, but are also markers of extra-embryonic endoderm (XEN), which arise from ESC undergoing ND or SFD. Mouse and human ESCs differentiated to XEN increase TMPRSS2 and other Covid-19 uptake-mediating gene expression, but only some lines express ACE2. Covid-19 susceptibility appears to be genotype-specific and not ubiquitous. Of the 30 gene ontology (GO) groups for viral susceptibility, 15 underwent significant stress-forced changes. Of these, $4 \mathrm{GO}$ groups mediated negative viral regulation and most genes in these increase in ND and decrease with SFD, thus suggesting that stress increases ESC viral susceptibility. Taken together, the data suggest that a control hyperosmotic stress can increase Covid-19 susceptibility and decrease viral host resistance in mouse ESC. However, this limited pilot study should be followed with studies in human ESC, tests of environmental, hormonal, and pharmaceutical stressors and direct tests for infection of stressed, cultured ESC and embryos by Covid-19.
\end{abstract}

Keywords High throughput screens $\cdot$ Embryonic stem cells $\cdot$ Hyperosmotic stress $\cdot$ Covid

Daniel A. Rappolee

drappole@med.wayne.edu

1 Department of Ob/Gyn, Reproductive Endocrinology and Infertility, CS Mott Center for Human Growth and Development, WayneState UniversitySchoolofMedicine, Detroit, Michigan 48201, USA

2 Reproductive Stress 3M Inc, Grosse Pointe Farms, MI 48236, USA

3 Washington University of St Louis, St. Louis, Missouri, USA

4 Reproductive Endocrinology and Cell Signaling LaboratoryDepartment of Integrative BiosciencesCollege of Veterinary Medicine and Biomedical Sciences, Texas A\&M University, College Station, Texas 77843, USA
5 Genome Sciences Center, Wayne State University School of Medicine, Detroit, MI 48201, USA

6 Institutes for Environmental Health Science, Wayne State University School of Medicine, Detroit 48202, USA

7 Program for Reproductive Sciences and Department of Physiology, Wayne State University School of Medicine, Detroit, MI 48201, USA

8 Invia Fertility Clinics, Hoffman Estates, Illinois 60169, USA

9 Department of Biology, University of Windsor, Windsor, ON N9B 3P4, Canada

10 CS Mott Center for Human Growth and Development, Wayne State University School of Medicine, 275 East Hancock, Detroit, MI 48201, USA 


\section{Introduction}

The Covid19/Sars-CoV-2 pandemic has caused trillions of dollars of economic disruption. World-wide, Sars-CoV-2 has infected an estimated 156 million people and Covid19 is implicated in more than 500,000 deaths in the U.S. alone (https://covid. cdc.gov/covid-data-tracker/\#cases_casesper100klast7days). Key elements of a successful Covid19 clinical responses are testing, tracking, reducing symptoms, vaccination, and risk analysis of viral effects dependent on susceptibility. An important element of risk analysis is whether environmental toxicants, urban hormonal stresses and nutrition affect susceptibility. Stresses have been linked in a dose-dependent manner to susceptibility through infection under circumstances where host responses and symptoms play a lesser role [1].

Covid 19 is particularly alarming as the newest of 3 coronaviruses since 2003 are the most severe: SARS1/Covid1, MERS and the current Covid19/Covid2/SARS2. Coronaviruses have unique dangerous capabilities that maintain pathogenic genomes during replication: the ability to swap genetic sequences, a large genome of $30 \mathrm{mb}, 3$ open reading frames producing 29 proteins, and genomic proof reading [2] during coinfection of host cells [3]. CoV2 has evolved changes that make it more clinically dangerous than previous coronaviruses. For example, it can infect upper respiratory throat and bronchi and create flu like symptoms or infect lungs and create pneumonia and death. Of the 7 total and 3 severe human coronaviruses (Cov1, Cov2, MERS), only Cov2 infects both the upper respiratory tract and lungs.

It is not surprising that host variation in susceptibility is a lesser focus, but it is still important to understand whether environmental stimuli change host susceptibility including virus uptake and interactome variation. This is especially dangerous in utero where early first trimester, placental syncytiotrophoblasts coexpress TMPRSS2 and ACE2 [4, 5], which are both required for cellular uptake and potential transplacental transfer of virus. Interestingly, it has been reported that human syncytiotrophoblasts (outer placental cells facing maternal circulation) and viruses do not co-express TMPRSS2 and ACE2 in second and third trimesters [6] suggesting that early first trimester embryos/fetuses and their stem cells are more exposed to Covid19 than stem cells in later fetuses. In addition, the other decidual tissue, extraembryonic endoderm, is also thought to co-express TMRPSS2 and ACE2, where co-expression is necessary for Covid 19 virion uptake $[4,7]$. Thus, the two membranes between the embryo/fetus and maternal virus, may both take up the virus and expose embryonic stem cells and/or derivative differentiated lineages. If stress increased Covid19 susceptibility genes and decreased viral resistance genes, lytic cell death in the early embryo could cause loss before detection of placental hormones and would not be recorded as a miscarriage. Occult losses or the stress-induced epigenetic memory of survival of early ESC lineage cells could lead to lineage imbalance or epigenetic error affecting the life of the placenta, yolk sac and neonate.

From the host cell perspective, a small number of genes is needed for cellular binding and uptake of the Cov2 virion. Throughout the host cells are protein interactomes, which can be targeting to enable drug repurposing, but also yield markers identifying susceptible cells [2, 8-10]. Key markers for susceptibility are a small set of genes binding receptors and fusigenic proteinases that clip the receptor and enable membrane fusion; cell surface and receptors - ACE2 human $\beta \operatorname{Cov} 1 / 2)$, Anpep and Anpep ( $\beta \operatorname{Cov} 2$ co-receptor candidate) [11], DPP4 (MERS $\beta$ Cov), and fusigenic serine proteases; TMPRSS2 ( $\beta \operatorname{Cov} 1 / 2)$, Cathepsins b/l (Ctsb, Ctsl), Furin $(\beta \operatorname{Cov} 1 / 2), \operatorname{Vim}[12]$.

There is a general knowledge of viral susceptibility genes, but also specific reports that ESC have antiviral responses and several groups of genes are necessary for these responses; histone chaperones, sumoylation factors, and chromatin modifiers which prevent proviral transcriptional activity [13, 14]. It is important to test whether known ESC viral resistance genes change expression during normal or stressed stemness or early differentiation. Gene ontology groups are a means to test host viral interactions, especially negative regulation, or resistance by host, in larger interacting gene sets [15].

We report here that nearly all these markers increase with normal ESC differentiation and increase further still with stress, and that viral resistance (i.e., negative regulatory) gene GO Groups increase with normal differentiation but decrease with stress. Taken together these transcriptomic changes, if accompanied by proteomic changes, would lead to greater viral infection in the earliest cells in the ESC lineage, probably XEN lineage cells, which are essential for embryonic survival by the start of gastrulation, two days after the embryo implants into the uterus [16].

\section{Materials and Methods}

\section{Materials}

Germline competent mESC-D3 cells were purchased from ATCC (Manassas, VA). DMEM medium was obtained from HyClone (Logan, UT). Gibco ${ }^{\mathrm{TM}}$ glutamax and sodium pyruvate supplement solutions were from Life Technologies (Grand Island, NY). ESC-qualified EmbryoMax fetal bovine serum, $0.1 \%$ gelatin solution and $\mathrm{ESGRO}^{\mathrm{TM}}$ Mouse LIF medium supplement were from EMD Millipore (Billerica, MA). Rex1 promoter reporter that enables expression of red 
fluorescent protein mApple were from Allele Biotechnology (San Diego, CA) as described previously [17, 18]. MEM nonessential amino acid solution, sorbitol, 2-mercaptoethanlol and other chemicals were from Sigma (St. Louis, MO). QIAzol Lysis reagent for mRNA isolation was from QiaGen (Ann Arbor, MI).

\section{Embryonic Stem Cell Culture and RNA Preparation}

Rex1-RFP embryonic stem cells were cultured as described previously [17, 18]. Rex1-RFP ESCs were optimized at passage for exponential growth during the stimulus period, which began $18 \mathrm{hr}$ after passage at $24 \%$ confluence. Germline competent mESC-D3 cells (ATCC, Manassas, VA) from Doetschman et al [19] were cultured in the absence of feeder cells in DMEM (Gibco, Grand Island, NY) supplemented with $15 \%$ mESC-screened fetal bovine serum (HyClone, Logan, UT), 2mM Glutamax, $1 \mathrm{mM}$ sodium pyruvate, 1 $\mathrm{mM}$ nonessential amino acids, $0.1 \mathrm{mM}$ 2-mercaptoethanol (Sigma, St. Louis, MO), and $1000 \mathrm{U} / \mathrm{mL}$ murine leukemia inhibitory factor (LIF; Millipore, Temecula, CA) on $0.1 \%$ gelatin-coated dishes at $37{ }^{\circ} \mathrm{C}$ in humidified air with $5 \%$ $\mathrm{CO} 2$ [20]. mESCs were cultured overnight after passaging before stimulation with sorbitol. Osmolality of ESC media with and without added $200-300 \mathrm{mM}$ sorbitol was determined previously [21]. ESC were washed at Tfinal and lysed using Trizol.

\section{RNA Isolation, cDNA Library Prep, and RNAseq}

RNA expression analysis was done by the Wayne State University Applied Genomics Technology Center with the following method. An aliquot of the RNA was assessed by microfluidics using the ScreenTape for the Agilent 2200 TapeStation. The electrophoretogram, RNA Integrity Number (RIN), and the ratio of the 28S:18S RNA bands were optimized for overall quality of the RNA as done previously. RNA-seq was used to determine expression profiles. Lexogen's QuantSeq 3'mRNA-seq Library Prep Kit (FWD for Illumina) was utilized for build ing RNA-seq libraries from 0.1-200 ng of total RNA in 5 $\mu$ l of nuclease-free ultrapure water. Libraries was quantified on the Qubit and Agilent 2200 Tapestation using the DNA High Sensitivity Screen tape. The barcoded libraries were multiplexed at equimolar concentrations and sequenced on an Illumina NovaSeq 6000. Data were demultiplexed using Illumina's CASAVA 1.8.2 software. After quality was assessed [22], and reads were aligned to the mouse genome (Build $\mathrm{mm} 9$ ) [23] and tabulated for each gene region [24]. Differential gene expression analysis was used to compare transcriptome changes between conditions [25]. Significantly altered genes (log fold change $\geq 2$; FDR $\leq 0.05$ ) were used to identify affected pathways [26].

\section{Statistics}

Data from at least three independent biological experiments were analyzed using Microsoft Excel and presented as means \pm SEM. Statistical analysis was done by student $t$-test. Geometric means analysis was used in the fluorescent flow cytometry study; the brightness of the fluorescent labeled cells ranges from low-to-high over a logarithmic range of fluorescence axis. This feature of fluorescent flow cytometry fits this criterion of geometric mean quantification, where higher reliability and repeatability were achieved than arithmetic mean quantification [27].

\section{Results}

The experimental protocol used a 72hr stimulus of ESC to normal stemness (NS) with stemness and proliferation-maintaining Leukemia inhibitory factor (LIF), normal differentiation by LIF removal (ND), and three levels of hyperosmotic stress as a positive control of stress-forced differentiation (SFD) used in previous studies (Figure 1 [18, 21, 28]). The 72hr stimulus was previously established for use with Rex1-RFP $[17,18]$ and Pdgfra-GFP [28] transgenic ESCs as it enables some differentiation and a 2-3fold change in stemness and 1st differentiated lineage, respectively. Doses of 200, 250 and $300 \mathrm{mM}$ sorbitol creates reduced growth, near zero growth, and negative growth, respectively. But, at highest dose initial death occurs only in the first $12 \mathrm{hr}$ and by $72 \mathrm{hr}$ there is approximately $5 \%$ death (data not shown, [21]), enabling assay of robust levels of stress responses where highest stimulus-response is induced. Comparisons are first used to test for significant and high fold change (FC) differences between ND from NS and then how SFD differs from ND. The final comparison is between SFD and NS to test how stress induces changes from the starting state of NS or naïve pluripotency equivalent to the source of ESC in the inner cells mass of the preimplantation embryo within 4.5 days of fertilization [29-32].

In Fig. 2A, the majority of XEN 1st differentiated lineage genes from two previous reports $[33,34]$ increased in cultured ESC here with ND (green box) with LIF removal but also increased more during exposure to $300 \mathrm{mM}$ sorbitol mediated SFD (red box) despite LIF. All other lineages reverse directions between increase in ND/NS and decrease in SFD/ND. Originating 0th lineage/Naïve pluripotency decreased with $\mathrm{ND} / 2$ nd lineage (to primarily formative pluripotency data not shown [35]) and increased with SFD. Also, primed pluripotency/3rd lineage increased with ND and decreased with SFD. In Fig. 2B, most genes in 4 different viral GO group processes increase during ND compared with NS and decrease during SFD compared with ND. In Fig. 2C, the majority of Covid19 or Covid1/MERS receptor genes increase with SFD compared with ND and ND more than NS in a manner like 1st lineage XEN increase in 
Fig. 1 Paradigm: 72hr exposure of embryonic stem cells (ESC) - grown in culture as stem cells - will identify stress levels that override growth and stemness and create teratogenic and epigenetic changes that persist and affect health. Terminology: we compare three states of ESC - normal stemness (NS) normal differentiation (ND) and the override; stress-forced differentiation (SFD). SFD is given at three doses that create normal growth to negative growth at this highest dose of positive control hyperosmotic. + control stress is hyperosmotic sorbitol at three doses
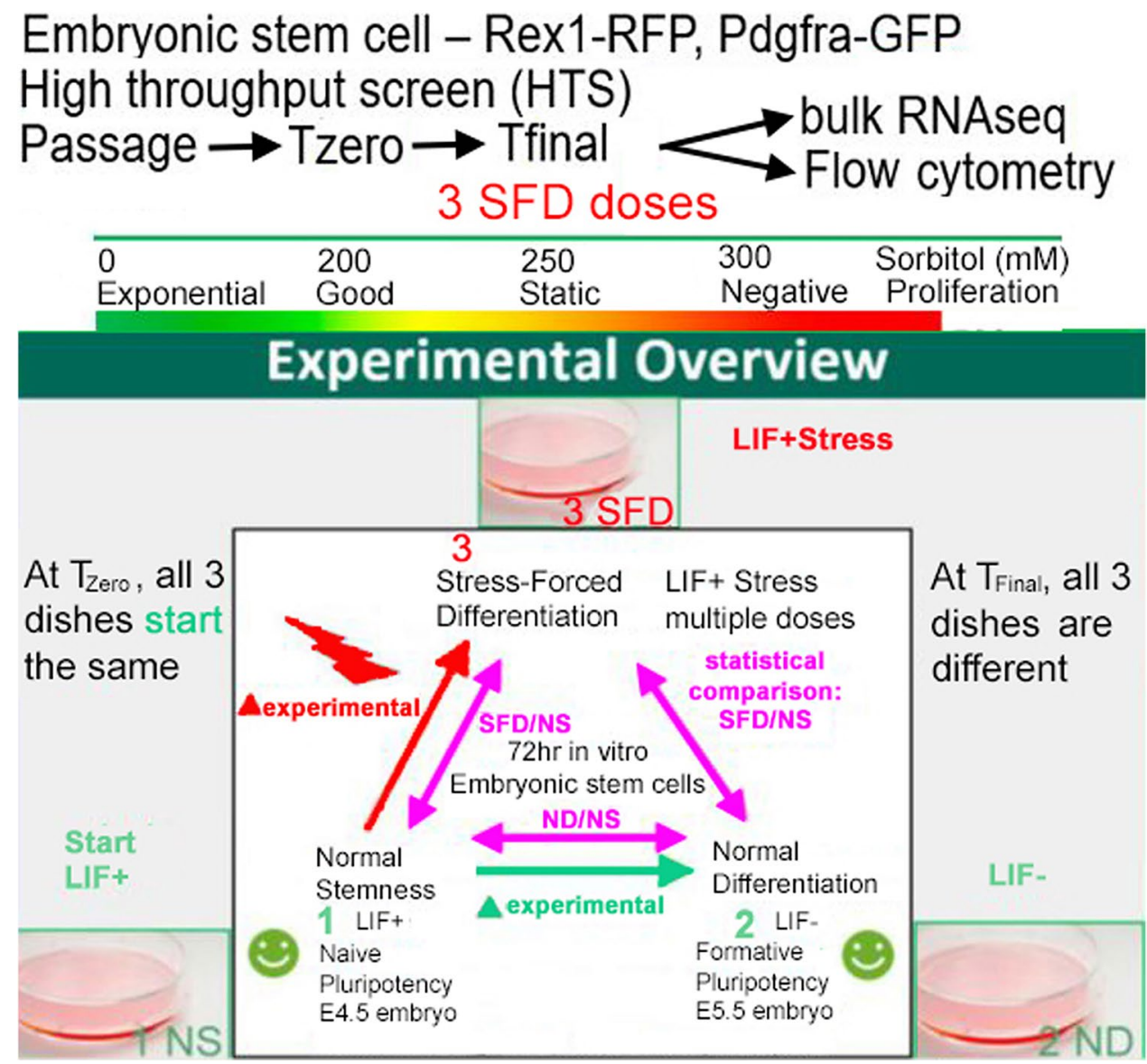

Fig. 2A. Most single viral susceptibility genes increase with ND and even more with SFD. Since TMPRSS2 is specific to endoderm and increases with ND and SFD, this suggests that stress may increase viral susceptibility by transdifferentiating ESC to XEN despite LIF presence. Fig. 2D shows the three sequential stages of embryogenesis, before and after implantation, and the lineage number assignments in temporal sequence as naïve pluripotency restricts to formative and then primed pluripotency at the start of gastrulation.

In Fig. 3 basal counts for coronavirus susceptibility genes in ESC culture under NS/naïve conditions tend to be at low levels, as for most transplacental viruses, suggesting low susceptibility in stem cells under naïve pluripotent culture, but a possible expression in subpopulations of cells in heterogeneous culture. Only one gene, Vim is expressed above 10counts in the Coronavirus receptor/ susceptibility gene group, whereas 3 genes, Pvrl1, Pvr and $\mathrm{Hn} 1$ are expressed above 10counts in transplacental viruses. After LIF removal for $72 \mathrm{hr}$ as ESCs begin the transit from Naïve pluripotency (NS) to Formative pluripotency (ND) and other subpopulations arise, most viral susceptibility genes upregulate (10/15) but no gene expression FC are significant. However, comparing ND to SFD highest dose at $300 \mathrm{mM}$ sorbitol stress $8 / 15$ genes have significant FC by Pvalue and/or FDR 10/15 genes upregulate. All 3 of the host coronavirus susceptibility genes that are significant in SFD FC are upregulated (Tmprss2, VIM, DPP4) and $2 / 5$ of the other genes, mostly transplacental virus susceptibility genes are upregulated (Havcr1, Pvrl1).

We analyzed total counts of mRNA for 20 viral receptors [36] Chapter on Biology of Viruses and Vira Diseases James D. Chappell and Terence S. Dermody] [36-39] in ESCs cultured under 1) NS conditions and then FC changes from NS to 2) ND or 3) $300 \mathrm{mM}$ sorbitol highest SFD to elucidate viral susceptibility under these three conditions. Supplemental Table 1. Eight of twenty viruses had $>10$ mRNA counts/cell during NS culture. In rank order these were the GNB2L1 > Rpsa $>$ Ldlr $>$ Pvrl2 > Cul5 > Icam1 > vim > Pvrl1 genes which are receptors for these viruses, respectively; influenza A $>$ Sindbis $>$ Rhino minor sero group $>$ herpes $2>$ encephalomyocarditis $>$ Rhino major sero group $>$ Covid $2>$ Herpes 1 . The highest upregulated genes/viral receptors with ND were CR2/Epstein Barr and Anpep/ several mammalian corona viruses. The highest downregulated genes/viral receptors with ND were CD4/HIV and Tnfrsf23/Pseudorabies. But the FC for all up- and 


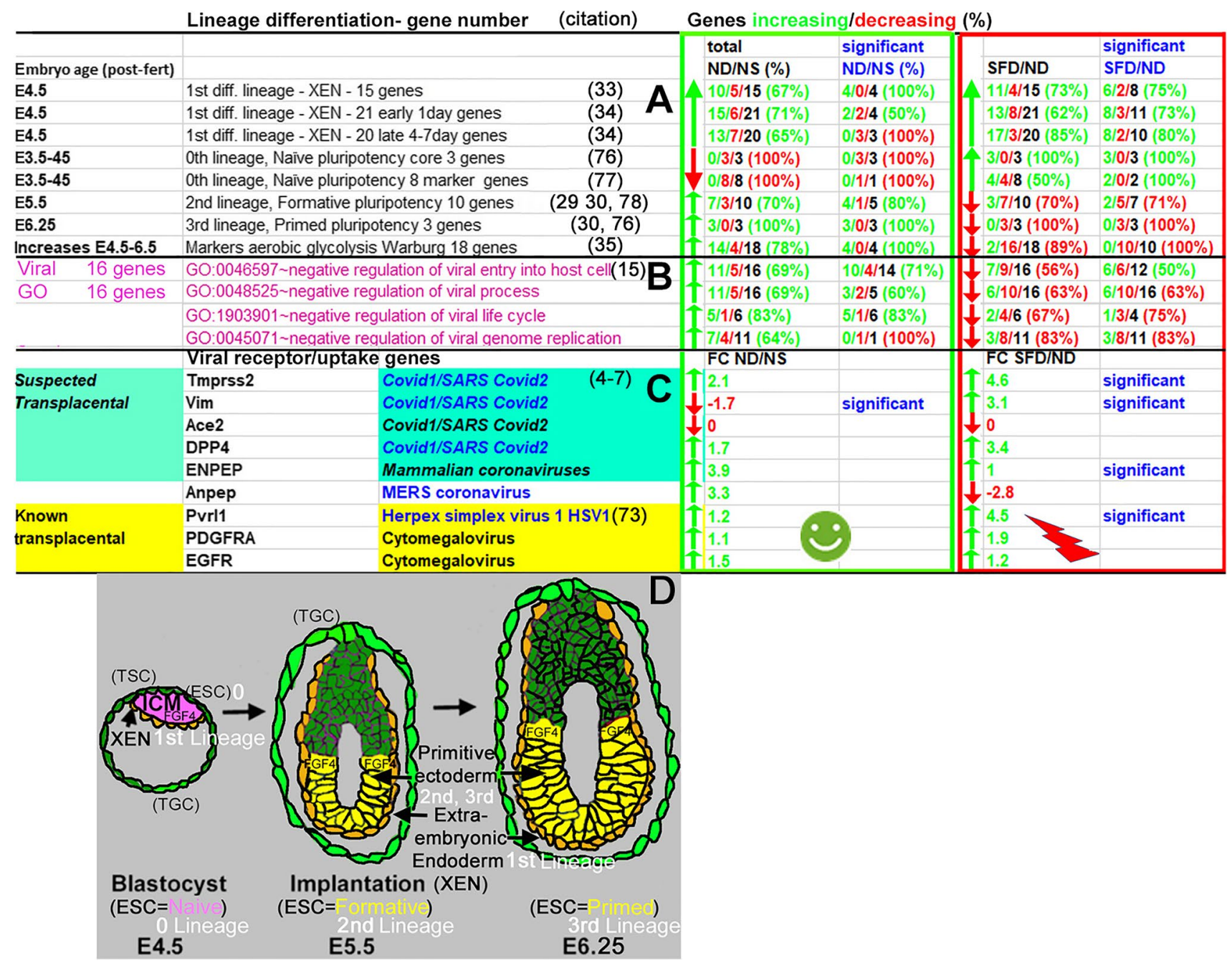

Fig. 2 Functional developmental gene groups (A) and GO group gene expression (B) are affected during normal differentiation without stress and stress-forced override of stemness at $300 \mathrm{mM}$ sorbitol, and single Viral susceptibility gene expression $(\mathbf{C})$ as described in Fig. 1. At the left are gene or gene group names. On the right, are fold changes in green (increase) or red (decrease) between ND from NS and on the far right SFD at 300mM Sorbitol compared to ND. (A) On the right total genes/group are black and decreasing and increasing genes are in red and green, respectively. The 8 developmental marker genesets arising in Embryos from E3.5-6.5 in vivo and in ESC in vitro from previous cited studies (in parentheses); 1)-[76] 3 core"0" or initiating naïve pluripotency (NS) genes downstream of LIF receptor: Stat3, Klf4, Tbx3 with 5 other naïve genes Tcf3, Nanog Essrb,Pou5F1, and an additional 2 Gjb5, Scpep1 [77] 2) ten 2nd lineage formative pluripotency genes: Dusp6, rip6, Lin28b [77], Otx2, Zic2 [78] CD47, Sox11, Egr1, Dnmt3b[29, 30, 79, 80], 3) Epha4a 3-three 3rd lineage "primed" pluripotency Sema6a, Car14, Jakmip2 [30, 76] 4) 21 1st lineage early XEN genes; Amn, Cd63, Ctsl, Fbp2, Gpx3, Lama1, Gsn, Lpar3, Lrpap1, Man2c1, P4ha2, PGK1, Col4a1/2, Podxl, S100a10, Serpinh1, Slc2a3, Sparc, Srgn, Tfp1 [34] 5) 20 1st lineage late XEN genes; Amn, Cd63, Ctsl, Clu, Ctsh, Lama1, EPAS1, Fst,Gata4/6, Lamb1, Lrpap1, P4ha2, Ph4b, Col4a1/2, Pga5, Serpinh1,,Sparc, Upp1 [34]) 6) 15 1st lineage XEN genes AFP, Gata4,
Sox17, Lamb1, Lama1, Aqp8, Vim, Plac1, Rhox6/9, Emp2, TROMA/ krt8, Col4a1/2, Amn [33]), for lineages 0/1/2/3, and 7) ghteen Aerobic glycolysis and Warburg genes; Slc2a1/Glut1, HK1, Pfkfb3, PGI-GPI, PFK1, Aldoa/c, Tpi1, Gapdh, PGK1,PGAM1, ENO1, PKM2, LDHA/B, PDH1, PDK1, SLc16a3-MCT4 [35] Starting "0" ESC lineage (E3.5-4.5) is Naïve pluripotent stem cells or NS from Fig. 1, 1st lineage from NS pluripotency is extraembryonic endoderm/ XEN and later lineages are restrictions of Naïve stemness: 2nd lineage formative pluripotency (E5.5) and 3rd lineage primed pluripotency. (B) The majority of genes in 4 viral negative regulatory groups increase from NS to ND, blocking viral pathogenesis, but gene expression in most genes in these groups reverses with SFD $300 \mathrm{mM}$ sorbitol compared with ND. (C) 6 coronavirus and 3 known transplacental susceptibility genes were studied, and upregulation or downregulation are indicated by fold change and significance by Pvalue and FDR. (D) A diagram of pre-implantation blastocyst at E4.5 (4.5 days after fertilization) and post-implantation embryo at E5.5 and near the start of gastrulation at E6.25. In the terminology of this figure, the 0th lineage is ICM/ESC at E4.5 which is naïve pluripotency/NS, 1st lineage is XEN arising before implantation, 2nd lineage is $\mathrm{ND} /$ formative pluripotency and 3rd lineage is primed pluripotency just before the start of gastrulation 


\begin{tabular}{|c|c|c|c|c|c|c|}
\hline Naïve $p$ & ripotenc & & Formativ & e pluripotency & & \\
\hline Normal st & emness & & Normal d & ifferentiation & & \\
\hline Viral & NS & & Viral & ND vs NS & & \\
\hline Receptor & Counts & & Receptor & & & \\
\hline Gene & $x$ & SD & Gene & Fold Change & PValue & \\
\hline Tmprss2 & 1 & 1 & Tmprss2 & 2.087582525 & 0.27992 & 1 \\
\hline Vim & 41 & 18.2 & Vim & -1.69560349 & 0.06753 & 1 \\
\hline Ace2 & 0 & 0 & Ace2 & -1 & 1 & 1 \\
\hline DPP4 & 2 & 1 & Dpp4 & 1.682 & 0.41625 & 1 \\
\hline Anpep & 1 & 0.5 & Anpep & 3.263007828 & 0.22242 & 1 \\
\hline ENPEP & 0 & 0 & Enpep & 3.861329928 & 0.25606 & 1 \\
\hline aver1 & 0 & 0 & Havcr1 & -1 & 1 & 1 \\
\hline vrl1 & 10 & 4.4 & Pvrl1 & 1.169125638 & 0.6763 & 1 \\
\hline PDGFRA & 1.7 & 2.1 & Pdgfra & 1.071 & 0.92879 & 1 \\
\hline EGFR & 6 & 3 & Egfr & 1.5171 & 0.23639 & 1 \\
\hline $\mathrm{Hn} 1$ & 291 & 176.7 & Hn1 & -1.04872437 & 0.80021 & 1 \\
\hline PVR & 19.1 & 8.2 & PVR & 1.177230264 & 0.55541 & 1 \\
\hline Mog & 0 & 0 & Mog & -1 & 1 & 1 \\
\hline pvrl4 & 1.7 & 0.6 & Pvrl4 & 2.605888289 & 0.15236 & 1 \\
\hline St3gal4 & 8 & 2.6 & St3gal4 & -1.51460822 & 0.31822 & 1 \\
\hline
\end{tabular}

Fig. 3 Stress changes expression for 6 host ESC genes for Coronavirus susceptibility, 8 genes for known transplacentally-transmitted virus susceptibility and 1 additional virus of highly significant changed susceptibility for virus uptake. These are single genes analyzed from bulk RNAseq for counts X+/-SD for ESC cultured under

down-regulated genes were not significant (pValue or FDR). The highest upregulated genes/viral receptors for 300mM sorbitol SFD were Havcr1/hepatitis A, Tnfrsf23/ Pseudorabies, Pvrl1/Herpes 1, and Vim/Covid2. Havcr1, Pvrl1, and Vim were all significant for $p$ Value and FDR. The highest downregulated genes/viral receptors for 300mM sorbitol SFD were CR2/Epstein-Barr and Cd4/ HIV, but none of these were significant.

Pdgfra-GFP ESC intermediate and bright subpopulations mark 1st differentiated lineage XEN cells, assayed by flow cytometry increased with increasing sorbitol above 300 to $400 \mathrm{mM}$ sorbitol, despite LIF presence (Fig. 4A). Only minor increases were observed in intermediate bright Pdgfra+ cells at $200-250 \mathrm{mM}$ sorbitol. Interestingly, $300 \mathrm{mM}$ sorbitol despite LIF produced almost equal increases in Pdgfra+ intermediate and bright cells as retinoic acid at $1 \mathrm{uM}$ with LIF removal an established inducer of XEN cells [28, 40]. Since retinoic acid at this level is a normal acid at $1 \mathrm{uM}$ with LIF removal an established inducer of XEN cells [28, 40]. Since retinoic acid at this level is a normal morphogen for XEN cells [40], this suggests that $300 \mathrm{mM}$ overrides LIF and forces differentiation of a subpopulation of ESC.

Stress increases Covid19 susceptibility genes that are markers of 1 st lineage XEN cells and their derivative extraembryonic endoderm lineages (Figs. 2, 3) and induces 1 st lineage as shown here and previously [21, 28, 41]. We thus surveyed mouse and human ESC and derivative XEN lineage differentiation at NCBI GEO profiles for expression of covid19 susceptibility genes TMPRSS2 and ACE2 and the XEN determining factor at the time of lineage allocation in the blastocyst, Gata6, which is essential in XEN lineage allocation in the blastocyst [42, 43]. All three genes were expressed at low or nil levels in undifferentiated embryonic
NS for $72 \mathrm{hr}$, and then FC (green increase and red decrease) for ND/ NS and for SFD highest dose at $300 \mathrm{mM}$ sorbitol/ND and then SFD highest dose/NS. Blue indicates significant FC between one culture treatment to another. On the far- right Green highlights indicate Coronavirus and yellow indicates trans-placentally-transmitted virus

stem cells but increase in some or all lineages of mouse or human XEN cells (Figure 4A, B). There was apparent heterogeneity in XEN differentiation and covid19 expression in different genetic backgrounds or due to other elements of quality of cell lines.

Of 15 significant changing host viral GO groups, 14 reverse most of their genes' expression from ND/ND to SFD 300mM/ND with 13 increasing with ND and decreasing with SFD and 1 decreasing with ND and increasing with SFD (Fig. 5). One GO group increased with ND and SFD. Rank of significance was higher during SFD $>$ ND for all 15 GO groups. Significance was rare for ND/NS and only 4/15 groups were significant changed but for SFD/ND all 15 groups changed significantly.

There are approximately 300 host viral interaction GO groups amongst approximately 45,000 groups (https://www. ebi.ac.uk/QuickGO/searchterms/viral, https://en.wikipedia. org/wiki/Gene_ontology\#Terms_and_ontology). In ESC, there were 92 genes in the 15 significant host viral response GO groups which are listed at the top of Supplemental Table 2. At the bottom of the table, are two auxiliary tables that show how the 92 genes are shared amongst all 15 groups and amongst the 4 negative regulatory groups. In the four negative regulatory groups the most common expressed genes in 3/4 groups are - jun, tetherin (aka Bst)2, and Interferoninduced transmembrane protein 1 (Ifitm1), and in $4 / 4$ groups are - 2'-5' -oligoadenylate synthetase 1 (Oas1b), Tripartite motif-containing 28 (Trim28), and Interferon-stimulated gene 15 (Isg15). Most of these have antiviral activities and some have known function in pluripotent cells. For all 15 groups the most commonly expressed genes are in 11/15 groups; oas $1 \mathrm{~b}$ and lsg15, in 9/15 groups; TRIM28, and in 7/15 groups; Krüppel-associated box zinc finger protein (Zfp)809, Ifitm1, 


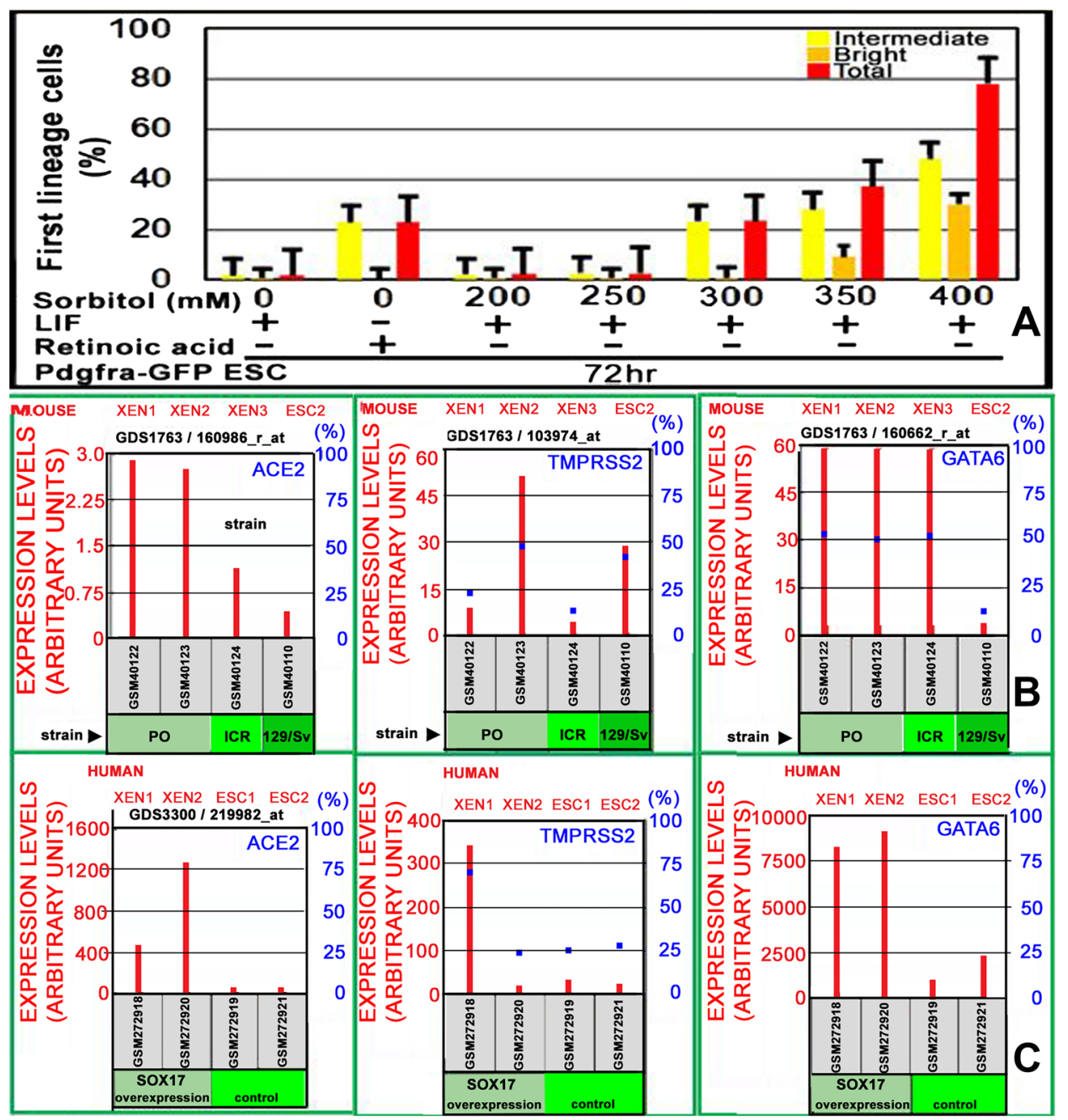

Fig. 4 First differentiated lineage XEN cells increase with $300 \mathrm{mM}$ sorbitol stress as much as positive control XEN morphogen retinoic acid, and mouse and human ESC differentiated to first lineage by culture conditions of Sox 17 transgenic overexpression, cause induction of ACE2 and Tmprss2 Covid19 susceptibility genes. (A) Pdgfra-GFP ESC were exposed to $72 \mathrm{hr}$ LIF+ (NS), LIF- (ND), $1 \mathrm{uM}$ retinoic acid a normal morphogen for 1 st lineage XEN induction from ESC or $200-400 \mathrm{mM}$ sorbitol in the presence of LIF to test for a stress dose that causes similar \% of bright and intermediate bright 1st lineage Pdgfra+ cells as assayed by flow cytometry [28], (B) Three mouse ESC lines were culture under conditions to main-

Nucleoporin 93 (Nup93), and nucleotide-binding oligomerization domain, leucine rich repeat containing X1 (Nlrx1).

At the other end of the expression spectrum are 22 genes expressed in only one GO group. Of these 22, 9 are of great interest because there is high counts and high and significant fold change in 2-3 of 3 possible comparisons: ND/ND or SFD/ND or SFD/NS. These 9 are Tmprss2, Proenkephalin (Penk), Basic leucine zipper transcription factor, ATFlike (Batf3), Myeloid differentiation primary response 88 (Myd88), Mitogen-activated protein kinase 11 (MAPK11, tain stemness (far right) or XEN differentiation (3 histogram bars on left) and tested for ACE2, Tmprss2 Covid19 susceptibility genes or Gata6 XEN determining transcription factor, from NCBI GEO database; GSM40110/22/23/24 [52]. (C) Two human ESC lines were culture under conditions to maintain stemness (2 histogram bars on far right) or two human ESC lines transdifferentiated to XEN differentiation using first lineage driver Sox17 ( 2 histogram bars on left) and tested for ACE2, Tmprss2 C ovid19 susceptibility genes or Gata6 XEN determining transcription factor, from NCBI GEO database; GSM2722918/19/20/21 [53]

aka p38MAPK $\beta$ ), heat shock protein beta-1 (Hspb1, aka HSP27), THO complex subunit 6 homolog (Thoc6), alphaactin-2 (Acta2), and Autophagy Related 16 Like 2 (Atg1612).

The highest dose at $300 \mathrm{mM}$ is a "demonstration dose" with highest fold change and significance, but so harsh in decreasing cell number during $72 \mathrm{hr}$ exposure, that this would be too morbid a dose to consider as affecting normal development. We thus assayed the lowest dose at $200 \mathrm{mM}$ sorbitol for which of the significant doses at $300 \mathrm{mM}$ were also affected significantly and found that only $5 / 15$ host viral response 
GO Group: change in expression of genes, significance rank of group, \% rank, and significance

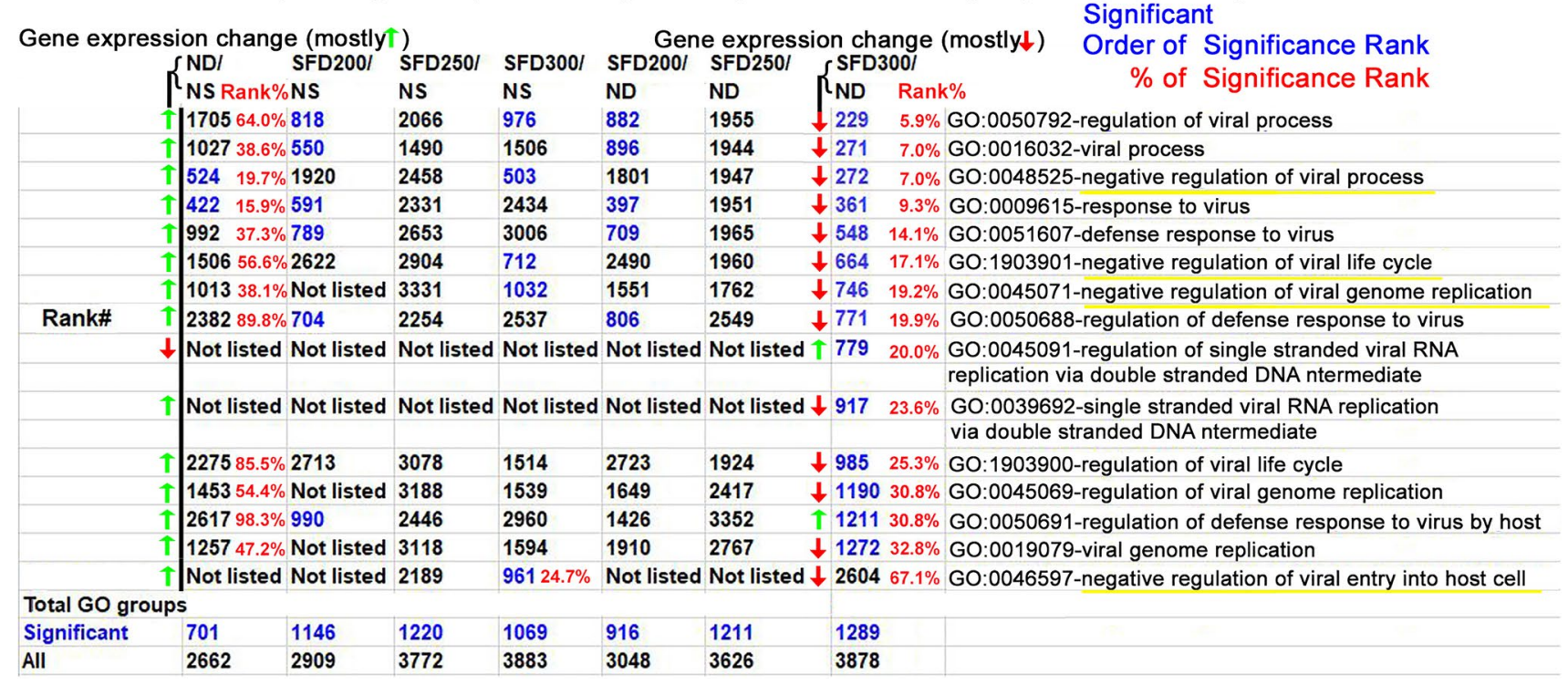

Fig. 5 Fifteen Viral GO groups were significant for stress induced fold changes in host ESC host-viral genome interactome genome rank of significance when comparing ND culture to NS culture but mostly SFD culture compared with NS or ND culture and 15 other viral host interactome GO Groups were not significant. 15 host viral GO groups for virus interaction had increasing significance at 300mM SFD compared with ND from top to bottom. Bottom two rows show total GO groups and significant GO groups for a comparison of culture ESC treatments
GO groups were affected. Of the GO groups significantly changed at $200 \mathrm{mM} 4 / 5$ are in the top 5 most significant. Interestingly, of the $15 \mathrm{GO}$ groups for host viral genes that had insignificance and lack of large change in ranking between unstressed and stressed groups at 300mM sorbitol (Fig. 5), the only directional regulatory were for 3 positive GO groups.

To confirm even expression of ESC cultured with 5 stimuli, 2 unstressed NS and ND (naïve and formative pluripotency, respectively), and 3 stressed $(200 \mathrm{mM}, 250 \mathrm{mM}$, and $300 \mathrm{mM}$ sorbitol) we tested for even expression with nonsignificant FC between stimuli and with low FC below 1.4. This was done by assaying the top twenty expressed genes at $300 \mathrm{mM}$ determined here and by using previous reported loading control [44-46] (Fig. 6A, B). For the top 20 highest expressed genes here, 13 are expressed at high stoichiometry in ribosomes, related to RNA loading controls in past studies of stress forced expression changes in mouse ESC [17, 18, 21, 28], but one of these had high and significant FC between NS and SFD (Fig. 6A). Of the 7 other highest expressed nonribosomal genes, only Tuba $1 \mathrm{~b}$ was also disqualified for high FC and significance. For more traditional loading controls, only Hprt was disqualified as a loading control for $\mathrm{FC}>1.4$ and significance, but several other genes had FC $>1.4$ without significance (Gapdh, PGK1, B2M, Ppia, Hmbs) (Fig. 6B). Altogether, the data from ribosomal, other highest expressed, and traditional loading controls suggest that total mRNA counts, and genes expressed per cell are well supported.

\section{Discussion}

We report here that single host viral susceptibility genes and several GO groups of host viral response and interaction genes, change with stress. This should increase viral Covid19 susceptibility specifically as well increasing susceptibility to as all viruses in general.

The culture model for ESC exposure used here replicated previous $72 \mathrm{hr}$ exposures used to test for stress effects on Rex1-RFP 0th lineage NS or naïve pluripotency loss [18] and Pdgfra-GFP 1st differentiated lineage XEN gain [28] and the sorbitol doses of 200, 250 and 300mM yield reduce but positive growth, near nil growth, and negative growth through initial apoptosis and chronic adaptation, respectively.

Most coronavirus susceptibility genes increase when NS differentiates to ND without stress by LIF removal and increase more when SFD at $300 \mathrm{mM}$ expression is compared with ND. In addition to increase to susceptibility due to single gene increases, the majority of all 4 negative regulatory $\mathrm{GO}$ groups increase with NS to ND, putatively suppressing viral processes. But most genes reverse and decrease expression with SFD compared with ND, suggesting that viral susceptibility due to stress-forced loss of resistance would exacerbate the coronavirus effects after entrance into the host cell. Taken together, stressed ESC express increased Covid receptors and decreased 
Fig. 6 Loading controls from highest copy number (A) and from previously published reviews (B). For ESD we typically use GAPDH, ACTB, and 18S loading controls which report high copy number RNA from ribosomes. Red shows decreasing expression from NS, green shows increasing expression from NS, pink shows ribosome-associated genes with names indicated in footnotes at the bottom, blue shows significance, orange shows order of ranking by highest expression in ESC at $300 \mathrm{mM}$ sorbitol and dark green is expression of the same genes as NS or $0 \mathrm{mM}$ sorbitol culture

\begin{tabular}{|c|c|c|c|c|c|}
\hline \multirow[t]{3}{*}{ LIF-I+ } & \multicolumn{2}{|c|}{ TOP 20 highest counts } & \multirow[t]{2}{*}{ SFD/ND } & NS & ORDER OF \\
\hline & & & & \multicolumn{2}{|c|}{ Pluripotency } \\
\hline & Fold Change & PValue & FDR & Corl Ave & Sorb 300 Ave \\
\hline Eir2s2 & -1.17 & 0.46 & 0.94 & 21307.33 & 16723.66 \\
\hline Aps 14 & 1.018 & 0.94 & 1 & 13802.66 & 13475.33 \\
\hline Hsp90ab1 & -1.28 & 0.27 & 0.74 & 18920.66 & 13423.33 \\
\hline Actb & 1.078 & 0.72 & 1 & 11941.33 & 12056.66 \\
\hline Ginb211 & -1.06 & 0.77 & 1 & 8837.66 & 8178.66 \\
\hline Pps 12 & 1.21 & 0.29 & 0.74 & 6849.66 & 8131.33 \\
\hline Rps20 & -1.3 & 0.14 & 0.58 & 11778.66 & 7536.33 \\
\hline Rpl23a & 1.01 & 0.95 & 1 & 7381.66 & 7371.33 \\
\hline Aps21 & 1.37 & 0.13 & 0.56 & 5350 & 7338.66 \\
\hline Aplp2 & 1.50 & 0.03 & 0.26 & 5135.66 & 7301 \\
\hline Apl8 & -1.25 & 0.29 & 0.74 & 9315.66 & 6570.66 \\
\hline Fus & 1. 12 & 0.68 & 1 & 5790.33 & 5653 \\
\hline Prds1 & 1.07 & 0.70 & 1 & 4900 & 5302 \\
\hline Aps3 & 1.22 & 0.31 & 0.76 & 4341.33 & 5260.33 \\
\hline Serbpt & 1.06 & 0.75 & 1 & 5261.33 & 5256 \\
\hline Hsp90aa1 & -1.49 & 0.08 & 0.46 & 8484.33 & 5154.33 \\
\hline Aplp1 & 1.33 & 0.26 & 0.73 & 3593.33 & 4931 \\
\hline Sod1 & -1.45 & 0.08 & 0.47 & 7761.33 & 4912.66 \\
\hline Rps5 & -1.28 & 0.22 & 0.72 & 6850.6 & 4894.33 \\
\hline Tuba1b & 2.17 & 0.00 & 0.02 & 2359 & 4853 \\
\hline
\end{tabular}

EIF2S2 is an intiatinge protein elongation factor that binds tPMA to 405 ribosomal subunits RPS are 405 structural ribosomal proteins

RPLP are 605 structural acidic proteins

RPL are 605 ribosomal structural proteins

GNB2L WRACK1 is a ribosomal 405 subunit binding protein

Serbp1 interacts with GNB2L IRACK1 and the ribosomal 405 subunit

\begin{tabular}{|c|c|c|c|c|c|c|c|c|c|c|c|c|c|c|c|}
\hline \multirow[b]{2}{*}{ Gene } & \multicolumn{3}{|c|}{ Normal stemness } & \multicolumn{4}{|c|}{ Normal differentiation ND/NS } & \multicolumn{4}{|c|}{ Stress-forced differentiation SFD/ND } & \multicolumn{3}{|c|}{ Stress-forced differentiation } & \multirow{2}{*}{$\begin{array}{l}\text { SFD/NS } \\
\text { e FDR }\end{array}$} \\
\hline & $\mathrm{x}$ & SD & gene & Fold Change & PValue & FDR & & gene & Fold Change & PValue & $F D R$ & gene & Fold Change & PValue & \\
\hline ACTB & 11941.3 & 7805.87 & Actb & -1.26 & 0.26 & 1 & reversal & Actb & 1.36 & 0.13 & 0.57 & Actb & 1.07 & 0.72 & 1 \\
\hline GAPDH & 130.7 & 177 & Gapdh & 2.24 & 0.12 & 1 & reversal & Gapdh & -1.60 & 0.36 & 0.81 & Gapdh & 1.40 & 0.51 & 0.99 \\
\hline Pgk1 & 19.7 & 22.9 & Pgk1 & 1.10 & 0.87 & 1 & reversal & Pgk1 & -2.00 & 0.29 & 0.73 & Pgk1 & -1.80 & 0.36 & 0.83 \\
\hline Ppia & 61.7 & 76.8 & Ppia & -1.24 & 0.70 & 1 & reversal & Ppia & 1.71 & 0.34 & 0.80 & Ppia & 1.37 & 0.57 & 1 \\
\hline RPLP0 & 2352.67 & 1389.73 & Rplp0 & 1.15 & 0.48 & 1 & reversal & Rplp0 & -1.19 & 0.38 & 0.84 & Rplp0 & -1.03 & 0.86 & 1 \\
\hline $\mathrm{B} 2 \mathrm{M}$ & 17.66 & 7.37 & B2m & 1.23 & 0.56 & 1 & reversal & $\mathrm{B} 2 \mathrm{~m}$ & -2.01 & 0.06 & 0.38 & B2m & -1.63 & 0.19 & 0.67 \\
\hline YWHAZ & 878.66 & 554.58 & Ywhaz & -1.19 & 0.38 & 1 & reversal & Ywhaz & -1.10 & 0.63 & 1 & Ywhaz & -1.31 & 0.17 & 0.64 \\
\hline Sdha & 258 & 98.7 & Sdha & 1.03 & 0.85 & 1 & reversal & Sdha & -1.12 & 0.53 & 1 & Sdha & -1.08 & 0.65 & 1 \\
\hline TFRC & 319.66 & 106.64 & Tfrc & 1.07 & 0.74 & 1 & reversal & Ifrc & -1.14 & 0.54 & 1 & Tfrc & -1.06 & 0.78 & 1 \\
\hline GUSB & 15 & 7.810 & Gusb & 1.06 & 0.82 & 1 & in kind & Gusb & 1.19 & 0.54 & 1 & Gusb & 1.27 & 0.40 & 0.87 \\
\hline HMBS & 28 & 12.76 & Hmbs & 1.37 & 0.24 & 1 & in kind & Hmbs & 1.04 & 0.88 & 1 & Hmbs & 1.43 & 0.18 & 0.66 \\
\hline HPRT & 15.66 & 1.52 & Hprt & -1.63 & 0.24 & 1 & in kind & Hprt & -2.29 & 0.08 & 0.43 & Hprt & -3.74 & 0.00 & 0.07 \\
\hline TBP & 16 & 1.73 & Tbp & 1.20 & 0.58 & 1 & in kind & Tbp & 1.13 & 0.70 & 1 & Tbp & 1.37 & 0.35 & 0.82 \\
\hline
\end{tabular}

negatively regulatory GO groups genes, which may be pathogenically synergistic. But does this occur in the stressed ESC themselves or in stress-forced differentiation to 1 st lineage.

Several lines of evidence suggest that XEN cells differentiated from ESCs may be the primary candidate cell for Covid19 infection. Several Covid19 genes are known to be expressed by XEN cells: TMPRSS2 [47] and DPP4 are expressed mostly by XEN visceral lineage [48, 49], ENPEP is expressed by mostly XEN [50], and VIM is expressed mostly by XEN parietal endoderm [51] . Thus, the induction of coronavirus is associated with the induction of XEN lineages. These covid susceptibility genes are expressed mostly at exceptionally low levels in the NS/ Naïve pluripotency starting state but increase with ND and more with SFD compared to NS. This suggests that low levels of these genes are expressed in naïve ESCs during
NS culture but an induction of a small subpopulation of 1 st lineage endoderm.

Flow cytometry was performed to test for stimulation induced increase from NS naïve pluripotency to ND formative pluripotency and to test for 1st subpopulation size increase in XEN. There was a large increase to an intermediate bright Pdgfra-GFP subpopulation of $\sim 20 \%$ and smaller bright subpopulation of $\sim 1 \%$ with $300 \mathrm{mM}$ sorbitol despite LIF and this was equivalent to the normal 1 st lineage XEN inducer retinoic acid with LIF removal [40, 49]. But a key deficit of induction of covid 19 susceptibility by stress was the lack of ACE2 in mouse ESC tested here in NS ND or SFD 300mM sorbitol. Both mouse and human ESC lines produce ACE2 and TMPRSS2 genes necessarily co-expressed for covid19 uptake after induction of XEN by cultural manipulation of mouse ESC [52] or transgenic overexpression of genes in human ESC, sox 7 or sox 17 
[53]. But not all mouse or human ESC lines expressed high levels of ACE2 suggesting genetic heterogeneity or some other deficit in inducing a full susceptibility interactome.

Stress increases significance of 15 viral host response GO groups and an increase in rank of significance from ND to SFD. Highest increases in rank are over 10fold from $64 \%$ to $5.9 \%$, but the most interesting are two that increase significantly from NS to ND and decrease significantly from SFD compared with ND. The least change is $47.2 \%$ to $32.8 \%$ (viral genome replication). but this is also a reversal from majority gene expression increase to majority decrease. Only one significant GO group does not undergo a reversal and it increases from 98.3 to $32.8 \%$ ranking from ND to SFD (regulation of defense of host to virus). Two GO groups are not listed in any pairwise comparison and are unranked but are highly significant, with $20.0 \%$ and $23.6 \%$, for SFD $300 \mathrm{mM}$ sorbitol compared to ND.

Six of the most shared genes are expressed in 3 or 4 of the four negative regulatory $\mathrm{GO}$ groups mediating viral resistance and $5 / 6$ of these genes have been reported to mediate viral resistance. Individual genes expressed in 3 of 4 of the negative regulatory host viral response GO groups are of great interest. Jun, Bst2 and Ifitm1 are expressed in three of the four groups. Jun is a stress transcription factor implicated in the stress response of ESC, TSC and early embryos. [54] Bst2, also known as tetherin is a protein associated with plasma-lemma lipid rafts and has been reported to inhibit retrovirus by preventing viral particles' diffusion into infected cells. [55] Ifitm $1 / 3$ is Interferon-induced transmembrane protein 1 . IFITM proteins are antiviral restriction factors for influenza A virus replication, probably at the early step of life cycle such as cellular entry and membrane fusion [56].

Individual genes expressed in 4 of 4 of the negative regulatory host viral response GO groups are also of great interest. Oas1b or 2'-5'-oligoadenylate synthetase 1 is an enzyme that is important in the innate immune response to viral infection [57]. Isg 15 or Interferon-stimulated gene 15 is a secreted cytokine and has intracellular function like ubiquitin, ISG15 is covalently linked by a C-terminal motif on newly-synthesized proteins, a process called ISGylation mediating both activation and inhibition of antiviral immunity [58] although viral resistance is mediated in mice but not humans [59]. Trim28 is in the tripartite motif (TRIM) protein family, which is a group of E3 ligases implicated in the regulation of a variety of cellular functions including innate immunity to virus which may occur by viral protein degradation in proteasomes and TRIM28 may block transition from latent to lytic cycle [60]. TRIM28 also has a role in maintaining pluripotency through epigenetic regulation [61] and is necessary to maintain epigenetic stability and imprinting during zygotic genome activation in mouse 2-cell stage embryos [62]. Although the phenotype is not the same as the ones studied here, the power of TRIM28 is reported in its role as the hierarchical epigenetic regulator of the complex polygenic, polyphenic cause of obesity, which TRIM28, depending on its wild type or haplo-insufficient activity, flips into an obese or non-obese state in isogenic animals that is dependent on the expression levels of a handful of imprinted genes [63]. TRIM28 is important in preventing provirus and endogenous retrovirus replication in ESC to protect germline lineage cells from insertional mutagenesis [13]. Interestingly, although most viral resistance genes decrease with stress, TRIM28 increases significantly with stress by both Pvalue and FDR (Supplemental Fig. S2). Covid-19 is not a retrovirus as it does not rely on reverse transcriptase and a DNA intermediate for replication. On the other hand some Cov2 sequence has been detected in host cells, suggesting that the RNA genome is reverse transcribed into DNA at low frequency and small parts integrate into the genome [64]. Taken together viral resistance is decreased by stress, and for some genes the amount of viral resistance function with stress is less than basal NS state as well as the ND state. It will be important to establish whether these genes affect viral resistance to coronaviruses as reported for their viral resistance function for other viruses and whether they are expressed and mediate function in XEN lineage cells. The specific mechanisms of viral resistance in ESC and the subpopulations of cells that are resistant or susceptible due to these mechanisms, need to be tested. It will be important to test whether Covid-19 can infect ESC and/or XEN cells and whether insertional mutagenesis can affect the germ line of ESC genomes.

Four genes common to 3-4/4 negatively regulatory GO groups are also highly shared amongst all 15 host viral GO groups, but Bst 2 and jun are not as highly shared. Genes highly shared amongst all 15 groups are: in 11/15 groups; oas $1 \mathrm{~b}$ and $\operatorname{lsg} 15$, in 9/15 groups; TRIM28, and in 7/15 groups; Krüppel-associated box zinc finger protein (Zfp809), Ifitm1, Nucleoporin 93 (Nup93), and nucleotide-binding oligomerization domain, leucine rich repeat containing X1 (Nlrx1). New genes common to all 15 but not most shared in 4 negative regulatory are ZFP809, Nlrx1, and Nup93. ZFP809 can mediate epigenetic anti-retroviral effects through histone modification more than DNA methylation after sequence recognition of foreign DNA [65]. Endogenous retroviruses are silenced primarily by DNA methylation in somatic tissue but by $\mathrm{H} 3$ methylation (H3K9me3) in embryos and ESC [66-68]. Nlrx1 found exclusively in the mitochondrial membrane, increases viral susceptibility by sequestering outer mitochondrial membrane proteins (MAVS) from another antiviral protein and thereby prevents mitochondrial antiviral immunity by preventing TBK1 to IRF3 to IFN $\beta$ transcription [69]. Nup93 is a nucleoporin that mediates mito viral immunity by preventing TBK1 to IRF3 to IFN $\beta$ transcription [69]. Nup93 is a nucleoporin that 
mediates mitochondrial activated TBK1 import to activate IRF3 and IFNB as part of innate immunity to retroviruses that Nlrx 1 blocks [70].

High stress at $300 \mathrm{mM}$ leads to high caspase 3 associated apoptosis in the first $12 \mathrm{hr}$ of stimulus but healthy cells with less than or about $5 \%$ death at $72 \mathrm{hr}$, whereas cell growth at $250 \mathrm{mM}$ is static and at $200 \mathrm{mM}$ significantly more cells accumulated at $72 \mathrm{hr}$ with a growth rate of about $75 \%$ at NS at $0 \%$ sorbitol [21]. At $200 \mathrm{mM}$ sorbitol only a third (5/15) of the GO groups significantly changed compared with all $(15 / 15)$ groups at $300 \mathrm{mM}$ sorbitol. As expected, four fifths (4/5) of the significantly changed GO groups are in the top 5 highest significant changed groups at $300 \mathrm{mM}$ sorbitol and are changed in the same direction at $200 \mathrm{mM}$. Thus, the quality and direction of the response at $200 \mathrm{mM}$ is similar that at $300 \mathrm{mM}$ sorbitol but is less significant and of lower ranking. Loading controls of several categories suggested that the conclusions for counts per cell for different genes, number of genes expressed, and GO groups genes were solid interpretations. It is likely that in vivo highest stress may come from exposures to multiple stressors that synergize to create stress like $300 \mathrm{mM}$ sorbitol or higher.

The greatest loss of human life occurs soon after fertilization, as the early embryo begins exponential growth and implants into the uterus to access maternal nutrition [54, $71,72]$. Just before implantation, embryonic stem cells and placental trophoblast stem cells arise in the embryo and have the capability of forming all 200+ cell types in adult humans and 10 lineages of the placenta, respectively. Viruses can harm the placenta or cross the placenta and infect the embryo or fetus: either of these events can have long term health risks for the fetus and offspring [73].

Key data suggest that maternal stress hormones sensitize the early post-implantation embryos and its ESC and TSC lineages to bisphenol A, leading to higher rates of miscarriage than either stressor alone [74]. Both types of stem cells in the implanting embryos respond to maternal stress hormones (adrenaline, cortisol) by growing more slowly [54] and other stressors such as benzopyrene of bisphenyl A slow growth and exacerbate stress hormone caused embryo loss in vivo [75]. Viral infectivity of 5 viruses was shown to be proportional to the amount of psychological stress and independent of other immune factors such as white blood cell counts [1], suggesting a focus stress-induced changes on viral receptors as a means to susceptibility. The data presented here support the hypothesis that stress can increase viral susceptibility by increasing receptor expression, but also suggest that stress can decrease host viral resistance, compounding Cov2 pathogenic potential in the early postfertilization embryo around the time of embryo implantation into the uterus. Direct testing whether ESC an their early differentiated progeny can be infected is needed, and whether this leads to lineage imbalance or genetic or epigenetic changes. It is also important to test whether environmental stressors, not just control hyperosmotic stress can change susceptibility of the ESC lineage.

\section{Conclusions}

A control hyperosmotic stress increases several Covid-19 viral receptor and susceptibility genes, and these genes are expressed at higher levels specifically in the stress-induced first differentiated lineage, extra-embryonic endoderm (XEN). Since XEN function is necessary for embryo survival during implantation into the uterus, viral infection may cause early miscarriage.

During normal or transgene-mediated induction of XEN in human and mouse ESC, Covid-19 receptor ACE2 is not always induced, suggesting possible genotype-specific Covid-19 susceptibility.

Stress also decreases the expression of the majority of genes in 4 host viral resistance gene GO groups during stress-forced differentiation, although the majority of genes in these groups increases with unstressed differentiation. Thus, one stress type can increase susceptibility and decrease host cell resistance. Since it has been reported that Covid-19 sequence has inserted into host genome by one lab, and another lab has shown that first lineage XEN can revert to totipotent early embryonic cells, transgenerational effects of viral insertions could be pathogenic. Thus, stress may increase immediate embryonic lethality and miscarriage, or long-term health effects after Covid-19 infection of cells arising from ESC soon after fertilization. This pilot study in mouse ESC should be extended to tests in human ESC, direct infection and possible genomic integration should be tested, and other stressors pregnant women are exposed to should be tested: hormonal stress, stress of environmental toxicants, diet supplements and pharmaceuticals.

Supplementary Information The online version contains supplementary material available at https://doi.org/10.1007/s12015-021-10188-w.

Acknowledgements Thanks to members of our lab for analysis and comments on the manuscript. Thanks to Summer interns Tanmai Nimmagadda and Munsir Jabir for analysis and assembly of Excel databases. Thanks to Dr William Robert Fleischmann, Jr and to Anthony Maxwell for comments on the manuscript Dr Rappolee is funded by NIH 1R41ES028991-01, 1R41ES031451-01A1, P30 ES020957/ CURES Pilot, and the Michigan Emerging Technology Fund. Dr. Ruden is funded by UH3 OD023285, S10 OD025170.

Authors' Contributions Mohammed Abdulhasan designed and performed experiments. Ximena Ruden contributed to writing and revising the manuscript. Benjamin Rappolee analyzed data and created figures. Sudipta Dutta contributed to experimental design and revising the manuscript. Katherine Gurdziel performed bioinformatic analysis. Douglas M. Ruden contributed to experimental design, funding, and revision of the manuscript. Awoniyi $\mathrm{O}$ Awonuga contributed to funding and 
revision of the manuscript. Steve J. Korzeniewski performed statistical analysis and revised the manuscript. Elizabeth E. Puscheck contributed to experimental design, funding, and revision of the manuscript. Daniel A. Rappolee contributed to experimental design, funding and writing and revision of the manuscript.

Funding DAR: 1R41ES028991-01, 1R41ES031451-01A1, Michigan Emerging Technology Fund and 5P30ES02095707 (Pilot grant). DMR: UH3 OD023285, S10 OD025170and 5P30ES02095707 (Pilot grant).

\section{References}

1. Cohen, S., Tyrrell, D. A., \& Smith, A. P. (1991). Psychological stress and susceptibility to the common cold. New England Journal of Medicine, 325, 606-12.

2. Gordon, D. E., Jang, G. M., Bouhaddou, M., et al. (2020). A SARS-CoV-2 protein interaction map reveals targets for drug repurposing. Nature, 583, 459-68.

3. Cyranoski, D. (2020). Profile of a killer: the complex biology powering the coronavirus pandemic. Nature, 581, 22-6.

4. Weatherbee, B. A. T., Glover, D. M., \& Zernicka-Goetz, M. (2020). Expression of SARS-CoV-2 receptor ACE2 and the protease TMPRSS2 suggests susceptibility of the human embryo in the first trimester. Open Biology, 10, 200162.

5. Ashary, N., Bhide, A., Chakraborty, P., et al. (2020). Single-Cell RNA-seq Identifies Cell Subsets in Human Placenta That Highly Expresses Factors Driving Pathogenesis of SARS-CoV-2. Frontiers in Cell and Development Biology, 8, 783.

6. Pique-Regi, R., Romero, R., Tarca, A. L., et al. (2020). Does the human placenta express the canonical cell entry mediators for SARS-CoV-2? Elife 9.

7. Colaco, S., Chhabria, K., Singh, N., et al. (2020). Expression of SARS-CoV-2 receptor ACE2 and the spike protein processing enzymes in developing human embryos. arXIV.

8. Zhou, Y., Hou, Y., Shen, J., et al. (2020). A Network Medicine Approach to Investigation and Population-based Validation of Disease Manifestations and Drug Repurposing for COVID-19. ChemRxiv.

9. Wang, R., Simoneau, C. R., Kulsuptrakul, J., et al. (2020). Genetic Screens Identify Host Factors for SARS-CoV-2 and Common Cold Coronaviruses. Cell.

10. Gordon, D. E., Hiatt, J., Bouhaddou, M., et al. (2020). Comparative host-coronavirus protein interaction networks reveal pan-viral disease mechanisms. Science, 370.

11. Qi, F., Qian, S., Zhang, S., \& Zhang, Z. (2020). Single cell RNA sequencing of 13 human tissues identify cell types and receptors of human coronaviruses. Biochemical and Biophysical Research Communications, 526, 135-40.

12. Yu, Y. T., Chien, S. C., Chen, I. Y., et al. (2016). Surface vimentin is critical for the cell entry of SARS-CoV. Journal of Biomedical Science, 23, 14.

13. Yang, B. X., El Farran, C. A., Guo, H. C., et al. (2015). Systematic identification of factors for provirus silencing in embryonic stem cells. Cell, 163, 230-45.

14. Wu, X., Kwong, A. C., \& Rice, C. M. (2019). Antiviral resistance of stem cells. Current in Opinion Immunology, 56, 50-9.

15. Foulger, R. E., Osumi-Sutherland, D., McIntosh, B. K., et al. (2015). Representing virus-host interactions and other multi-organism processes in the Gene Ontology. BMC Microbiology, 15, 146-57.

16. Rappolee, D. A. (1999). It's not just baby's babble/Babel: recent progress in understanding the language of early mammalian development: a minireview. Molecular Reproduction and Development, $52,234-40$.
17. Li, Q., Yang, Y., Louden, E., Puscheck, E., Rappolee, D. (2016). High throughput screens for embryonic stem cells; stress-forced potency-stemness loss enables toxicological assays. In: Faqi A, ed. Methods In Toxicology and Pharmacology: Springer.

18. Li, Q., Gomez-Lopez, N., Drewlo, S., et al. (2016). Development and Validation of a Rex1-RFP Potency Activity Reporter Assay That Quantifies Stress-Forced Potency Loss in Mouse Embryonic Stem Cells. Stem Cells and Development, 25, 320-8.

19. Doetschman, T. C., Eistetter, H., Katz, M., Schmidt, W., \& Kemler, R. (1985). The in vitro development of blastocyst-derived embryonic stem cell lines: formation of visceral yolk sac, blood islands and myocardium. Journal of Embryology and Experimental Morphology, 87, 27-45.

20. Masui, S., Ohtsuka, S., Yagi, R., Takahashi, K., Ko, M. S., \& Niwa, H. (2008). Rex1/Zfp42 is dispensable for pluripotency in mouse ES cells. BMC Development Biology, 8, 45.

21. Slater, J. A., Zhou, S., Puscheck, E. E., \& Rappolee, D. A. (2014). Stress-induced enzyme activation primes murine embryonic stem cells to differentiate toward the first extraembryonic lineage. Stem Cells and Development, 23, 3049-64.

22. Andrews, S. (2010). FastQC A Quality Control Tool for High Throughput Sequence Data.

23. Dobin, A., Davis, C. A., Schlesinger, F., et al. (2013). STAR: ultrafast universal RNA-seq aligner. Bioinformatics, 29, 15-21.

24. Anders, S., Pyl, P. T., \& Huber, W. (2015). HTSeq-a Python framework to work with high-throughput sequencing data. Bioinformatics, 31, 166-9.

25. Robinson, M. D., McCarthy, D. J., \& Smyth, G. K. (2010). edgeR: a Bioconductor package for differential expression analysis of digital gene expression data. Bioinformatics, 26, 139-40.

26. Huang, W., Sherman, B. T., \& Lempicki, R. A. (2009). Systematic and integrative analysis of large gene lists using DAVID bioinformatics resources. Nature Protocols, 4, 44-57.

27. Wu, Y. J., Wang, H., Liang, J. H., et al. (2016). Using the geometric mean fluorescence intensity index method to measure ZAP-70 expression in patients with chronic lymphocytic leukemia. Oncotargets and Therapy, 9, 797-805.

28. Li, Q., Louden, E., Zhou, J., et al. (2019). Stress Forces First Lineage Differentiation of Mouse Embryonic Stem Cells; Validation of a High-Throughput Screen for Toxicant Stress. Stem Cells and Development, 28, 101-13.

29. Smith, A. (2017). Formative pluripotency: the executive phase in a developmental continuum. Development, 144, 365-73.

30. Kalkan, T., Olova, N., Roode, M., et al. (2017). Tracking the embryonic stem cell transition from ground state pluripotency. Development, 144, 1221-34.

31. Munoz Descalzo, S., Rue, P., Garcia-Ojalvo, J., \& Martinez, A. A. (2012). Correlations between the levels of Oct4 and Nanog as a signature for naive pluripotency in mouse embryonic stem cells. Stem Cells, 30, 2683-91.

32. Chen, H., Aksoy, I., Gonnot, F., et al. (2015). Reinforcement of STAT3 activity reprogrammes human embryonic stem cells to naive-like pluripotency. Nature Communications, 6, 7095.

33. Han, X., Wang, R., Zhou, Y., et al. (2018). Mapping the Mouse Cell Atlas by Microwell-Seq. Cell, 173, 1307.

34. Klein, A. M., Mazutis, L., Akartuna, I., et al. (2015). Droplet barcoding for single-cell transcriptomics applied to embryonic stem cells. Cell, 161, 1187-201.

35. Abdulhasan, M., Dutta, S., Gurdziel, K., et al. (2021). Validating a high throughput screen using embryonic stem cells: transcriptomic analysis identifies unique aspects of stress-forced cellular adaptation. Cells in Preparation.

36. Bennett, J. E., Dolin, R., Blaser, M. J. (2015). Mandell, Douglas, and Bennett's principles and practice of infectious diseases. Eighth edition. ed. Philadelphia, PA: Elsevier/Saunders. 
37. Barrass, S. V., Butcher, S. J. (2019). Advances in high-throughput methods for the identification of virus receptors. Medical Microbiology and Immunology.

38. Mestecky, J. (2005). Mucosal immunology (3rd ed.). . Amsterdam; Boston: Elsevier Academic Press.

39. Pillay, S., \& Carette, J. E. (2015). Hunting Viral Receptors Using Haploid Cells. Annual Review of Virology, 2, 219-39.

40. Artus, J., Panthier, J. J., \& Hadjantonakis, A. K. (2010). A role for PDGF signaling in expansion of the extra-embryonic endoderm lineage of the mouse blastocyst. Development, 137, 3361-72.

41. Abdulhasan, M., Dutta, S., Li, Q., et al. (2021). Validating a high throughput screen using embryonic stem cells: transcriptomic analysis identifies stress-forced developmental adaptation. Stem Cell Research in Preparation.

42. Morrisey, E. E., Tang, Z., Sigrist, K., et al. (1998). GATA6 regulates HNF4 and is required for differentiation of sceral endoderm in the mouse embryo. Genes and Development, 12, 3579-90.

43. Koutsourakis, M., Langeveld, A., Patient, R., Beddington, R., \& Grosveld, F. (1999). The transcription factor GATA6 is essential for early extraembryonic development. Development, 126, 723-32.

44. Moein, S., Javanmard, S. H., Abedi, M., Izadpanahi, M. H., \& Gheisari, Y. (2017). Identification of Appropriate Housekeeping Genes for Gene Expression Analysis in Long-term Hypoxiatreated Kidney Cells. Advanced Biomedical Research, 6, 15.

45. Curina, A., Termanini, A., Barozzi, I., et al. (2017). High constitutive activity of a broad panel of housekeeping and tissue-specific cis-regulatory elements depends on a subset of ETS proteins. Genes and Development, 31, 399-412.

46. Eisenberg, E., \& Levanon, E. Y. (2013). Human housekeeping genes, revisited. Trends in Genetics, 29, 569-74.

47. Yasunaga, M., Tada, S., Torikai-Nishikawa, S., et al. (2005). Induction and monitoring of definitive and visceral endoderm differentiation of mouse ES cells. Nature Biotechnology, 23, 1542-50.

48. Kinoshita, M., Shimosato, D., Yamane, M., \& Niwa, H. (2015). Sox 7 is dispensable for primitive endoderm differentiation from mouse ES cells. BMC Developmental Biology, 15, 37.

49. Artus, J., Douvaras, P., Piliszek, A., Isern, J., Baron, M. H., \& Hadjantonakis, A. K. (2012). BMP4 signaling directs primitive endoderm-derived XEN cells to an extraembryonic visceral endoderm identity. Developmental Biology, 361, 245-62.

50. Rugg-Gunn, P. J., Cox, B. J., Lanner, F., et al. (2012). Cell-surface proteomics identifies lineage-specific markers of embryo-derived stem cells. Developmental Cell, 22, 887-901.

51. Paca, A., Seguin, C. A., Clements, M., et al. (2012). BMP signaling induces visceral endoderm differentiation of XEN cells and parietal endoderm. Developmental Biology, 361, 90-102.

52. Kunath, T., Arnaud, D., Uy, G. D., et al. (2005). Imprinted $\mathrm{X}$-inactivation in extra-embryonic endoderm cell lines from mouse blastocysts. Development, 132, 1649-61.

53. Seguin, C. A., Draper, J. S., Nagy, A., \& Rossant, J. (2008). Establishment of endoderm progenitors by SOX transcription factor expression in human embryonic stem cells. Cell Stem Cell, 3, 182-95.

54. Puscheck, E. E., Awonuga, A. O., Yang, Y., Jiang, Z., \& Rappolee, D. A. (2015). Molecular biology of the stress response $\mathrm{n}$ the early embryo and its stem cells. Advances in Experimental Medicine and Biology, 843, 77-128.

55. Sakuma, T., Noda, T., Urata, S., Kawaoka, Y., \& Yasuda, J. (2009). Inhibition of Lassa and Marburg virus production by tetherin. Journal of Virology, 83, 2382-5.

56. Feeley, E. M., Sims, J. S., John, S. P., et al. (2011). IFITM3 inhibits influenza A virus infection by preventing cytosolic entry. PLoS Pathogens 7:e1002337-e.

57. Green, R., Wilkins, C., Thomas, S., et al. (2017). Oas1b-dependent Immune Transcriptional Profiles of West Nile Virus Infection in the Collaborative Cross. G3 (Bethesda) 7:1665-82.
58. Woods, M. W., Kelly, J. N., Hattlmann, C. J., et al. (2011). Human HERC5 restricts an early stage of HIV-1 assembly by a mechanism correlating with the ISGylation of Gag. Retrovirology, 8, 95-110.

59. Speer, S. D., Li, Z., Buta, S., et al. (2016). ISG15 deficiency and increased viral resistance in humans but not mice. Nature Communications, 7, 11496-506.

60. van Tol, S., Hage, A., Giraldo, M. I., Bharaj, P., \& Rajsbaum, R. (2017). The TRIMendous Role of TRIMs in Virus-Host Interactions. Vaccines (Basel), 5, 23.

61. Oleksiewicz, U., Gładych, M., Raman, A. T., et al. (2017). TRIM28 and Interacting KRAB-ZNFs Control Self- Renewal of Human Pluripotent Stem Cells through Epigenetic Repression of Pro-differentiation Genes. Stem Cell Reports, 9, 2065-80.

62. Messerschmidt, D. M., de Vries, W., Ito, M., Solter, D., FergusonSmith, A., \& Knowles, B. B. (2012). <em $>$ Trim $28</ \mathrm{em}>$ Is Required for Epigenetic Stability During Mouse Oocyte to Embryo Transition. Science, 335, 1499-502.

63. Dalgaard, K., Landgraf, K., Heyne, S., et al. (2016). Trim28 Haploinsufficiency Triggers Bi-stable Epigenetic Obesity. Cell, 164, 353-64.

64. Zhang, L., Richards, A., Khalil, A., et al. (2020). SARS-CoV-2 RNA transcribed and integrated into the human genome. bioRxiv.

65. Wolf, G., Yang, P., Füchtbauer, A. C., et al. (2015). The KRAB zinc finger protein ZFP809 is required to initiate epigenetic silencing of endogenous retroviruses. Genes and Development, 29:538-54

66. Mikkelsen, T. S., Ku, M., Jaffe, D. B., et al. (2007). Genome-wide maps of chromatin state in pluripotent and lineage- committed cells. Nature 448:553-60.

67. Rebollo, R., Romanish, M. T., \& Mager, D. L. (2012). Transposable elements: an abundant and natural source of regulatory sequences for host genes. Annual Review of Genetics, 46, 21-42.

68. Wolf, G., Yang, P., Fuchtbauer, A. C., et al. (2015) The KRAB zinc finger protein ZFP809 is required to initiate epigenetic silencing of endogenous retroviruses. Genes and Development, 29, 538-54.

69. O'Neill, L. A. (2008). Innate immunity: squelching anti-viral signalling with NLRX1. Current Biology, 18, R302-4.

70. Monwan, W., Kawasaki, T., Hasan, M. Z., Ori, D., \& Kawai, T. (2020). Identification of nucleoporin 93 (Nup93) that mediates antiviral innate immune responses. Biochemical and Biophysical Research Communications, 521, 1077-82.

71. Yang, Y., Bolnick, A., Shamir, A., et al. (2017). BlastocystDerived Stem Cell Populations under Stress: Impact of Nutrition and Metabolism on Stem Cell Potency Loss and Miscarriage. Stem Cell Reviews, 13, 454-64.

72. Yang, Y., Abdulhasan, M., Awonuga, A., Bolnick, A., Puscheck, E. E., \& Rappolee, D. A. (2017). Hypoxic Stress Forces Adaptive and Maladaptive Placental Stress Responses in Early Pregnancy. Birth Defects Research, 109, 1330-44.

73. Racicot, K., \& Mor, G. (2017). Risks associated with viral infections during pregnancy. The Journal of clinical investigation, 127, 1591-9.

74. Borman, E. D., Foster, W. G., Greenacre, M. K. E., Muir, C. C., \& deCatanzaro, D. (2015). Stress lowers the threshold dose at which bisphenol A disrupts blastocyst implantation, in conjunction with decreased uterine closure and ecadherin. Chemico-Biological Interactions, 237, 87-95.

75. Borman, E. D., Foster, W. G., Greenacre, M. K., Muir, C. C., \& deCatanzaro, D. (2015). Stress lowers the threshold dose at which bisphenol A disrupts blastocyst implantation, in conjunction with decreased uterine closure and ecadherin. Chemico-Biological Interactions, 237, 87-95.

76. Bernardo, A. S., Jouneau, A., Marks, H., et al. (2018) Mammalian embryo comparison identifies novel pluripotency genes associated with the naive or primed state. Biology Open, 7.

77. Martello, G., Sugimoto, T., Diamanti, E., et al. (2012). Esrrb is a pivotal target of the Gsk3/Tcf3 axis regulating embryonic stem cell self-renewal. Cell Stem Cell, 11, 491-504. 
78. Weinberger, L., Ayyash, M., Novershtern, N., \& Hanna, J. H. (2016). Dynamic stem cell states: naive to primed pluripotency in rodents and humans. Nature Reviews Molecular Cell Biology, $17,155-69$.

79. Kalkan, T., Bornelov, S., Mulas, C., et al. (2019). Complementary Activity of ETV5, RBPJ, and TCF3 Drives Formative Transition from Naive Pluripotency. Cell Stem Cell, 24(785-801), e7.
80. Kim, H. J., Osteil, P., Humphrey, S. J., et al. (2020). Transcriptional network dynamics during the progression of pluripotency revealed by integrative statistical learning. Nucleic Acids Research, 48, 1828-42.

Publisher's Note Springer Nature remains neutral with regard to jurisdictional claims in published maps and institutional affiliations. 